\title{
Interaction, Mediation, and Ties: An Analytic Hierarchy for Socio-Technical Systems
}

\author{
Daniel D. Suthers \\ Dept. of Information and Computer Sciences \\ University of Hawai' $i$ \\ suthers@hawaii.edu
}

\begin{abstract}
To understand how technological designs encourage synergistic encounters between people and ideas within socio-technical systems, techniques are needed to bridge between levels of description from process traces such as log data, through individual trajectories of activity that interact with each other, to dynamic networks of associations that are both created by and further shape these interactions. Towards this end, we have developed an analytic hierarchy and associated representations. Process traces are abstracted to contingency and uptake graphs: directed graphs that record observed relationships (contingencies) between events that offer evidence for interaction and other influences between actors (uptake). Contingency graphs are further abstracted to associograms: two-mode directed graphs that record how associations between actors are mediated by digital artifacts. Patterns in associograms summarize sequential patterns of interaction. Transitive closure of associograms yields sociograms, to which existing network analytic techniques may be applied. We discuss how the hierarchy bridges between theoretical levels of analysis.
\end{abstract}

\section{Introduction}

The rapid growth of socio-technical networkssocial networks embedded in communication and information technologies-and their pervasive integration into all areas of modern life is well known. As these networks scale up and new forms of technology-mediated social entities emerge, it becomes increasingly important to answer fundamental questions concerning how technological designs encourage synergistic encounters between and transformations of people and ideas within these networks. We need to understand when and where members participate and with whom; through what kinds of interactions relationships are formed and value created; what information is shared and knowledge is formed and sustained in these interactions; and how patterns of participation lead to the diffusion of ideational and digital resources. Furthermore, we need to understand how participants appropriate and are influenced by technological resources, and how relationships and processes change dynamically over time. These questions suggest that multiple theoretical perspectives be taken on socio-technical systems, and at different levels of analysis. We need to understand the interaction that takes place between actors and the work that gets done through that interaction. We also need to understand how this interaction is organized by and gives rise to communication networks in which ideas and influences flow between actors. Finally, we need to understand the mediating roles of digital artifacts that both offer resources for and are appropriated by participants for interaction in these networks.

The instrumental goal of this work is to develop a framework for modeling interaction, mediation and ties that will enable this multi-level understanding of socio-technical systems. Techniques are needed to bridge between levels of description from event log data, through individual trajectories of activity and how they intersect and affect each other, to the dynamic networks of associations that both are created by and further shape these interactions. We need tools that generalize across individual systems, are usable by both social and computing science researchers, and enable them to construct analytic representations that support the development of theories about technology mediation of social and intellectual life.

Towards this end, we have developed a hierarchy of analytic representations and associated tools to trace out the movement, confluences, and transformations of actors, artifacts and ideas. Our prior work developed the first level of abstraction from $\log$ files. Contingency graphs are directed graphs that record observed relationships (contingencies) between events that may be taken as 


\section{Table 1. The Analytic Hierarchy}

\begin{tabular}{ll} 
Level of Modeling & Representations \\
\hline Process Traces & Log files, audio and video recordings, etc. \\
\hline Domain & Entities and their relationships (types and instances of both) \\
\hline Event & Sets of Events (described in terms of their actors, objects, temporal indices, etc.) \\
\hline Contextualized Action & Contingency Graphs indicating empirical relationships (“contingencies") between Events \\
\hline Interaction & Uptake Graphs (each arc corresponds to bundles of contingencies that evidence uptake) \\
\hline Mediation & Associograms, or two-mode directed graphs relating actors to objects \\
\hline Relationships & Subgraphs of the Mediation model consisting of all paths between two actors \\
\hline Ties & Sociograms representing ties between Actors
\end{tabular}

evidence for interaction and other associations or influences between actors. Uptake graphs represent the sequential structure of interaction derived from contingency graphs. This paper extends this work to include another second-order representation that has been found useful for summarizing the roles of mediating artifacts in interaction, and that also bridges from interaction to network levels of description. Associograms are two-mode directed graphs that record how associations between actors are mediated by their creation, modification, and access of digital artifacts. Associograms can be automatically generated from contingency graphs. We later examine how patterns in associograms indicate sequential patterns of interaction. Thus they summarize more complex interaction data. Pathways between two actors in an associogram characterize the mediated nature of the relationship between those persons. Associograms can also be transformed to conventional sociograms by transitive closure of mediated associations. Thus they bridge between interaction data and networks to which existing network analytic techniques may be applied. This paper describes the analytic hierarchy, and discusses potential roles of this analytic hierarchy in bridging between levels of analysis and theory.

The analytic hierarchy consists of several abstraction layers of analytic representations, summarized in Table 1, that we have found to be useful for various specific analyses in our laboratory. Our analytic needs are diverse (we study technology mediated interaction ranging from dyads to online communities), so we were motivated to generalize these representations in a manner that may also meet others' needs. Beyond meeting our immediate research objectives, our motivations for constructing this analytic hierarchy include enabling the development of shared conceptualizations, representations, and tools at a given level of analysis and supporting bridging between different levels of analysis, both of which are necessary foundations for productive discourse in multidisciplinary fields such as the study of socio-technical networks.
The layers are explained in more detail in the following subsections. The lowest levels of abstraction (process trace, domain model, event model) and transformations between them are likely to be familiar to readers: brief sections on them are included only for completeness and to provide the foundation and examples for describing subsequent layers. Contingency and Uptake Graphs (contextualized action model and interaction model, respectively) are more unique contributions, so are described in more detail here-see also [21] for extensive discussion. The most attention will be given to Associograms (mediation models) and their relationships to the other layers, as this layer has not been described in detail elsewhere. The most abstract layer (social ties) is covered substantially in the social network analysis literature [e.g., 24], so is described here only in relation to how it is derived from the layer below, and what that vertical relationship enables that would not be possible with direct measurement of ties. After this presentation, implications for the integrated study of sociotechnical systems as communication networks are discussed.

\section{Process Traces}

Any analysis of interaction begins with a process trace, or record of activity left in the environment and accessible to the researcher. Examples include software log data (software application or server $\operatorname{logs}$ ), audio and video recordings, and textual transcripts. The analytic hierarchy described herein was designed to support analysis of both software logs and video recordings, sometimes in conjunction (e.g., we have analyzed application logs and screen capture of the same application [12, 13]). For simplicity of presentation and to emphasize the potential for automated analysis, this paper focuses on software logs, and does not touch on issues of video analysis; see [7, 8] for discussion of such issues. 


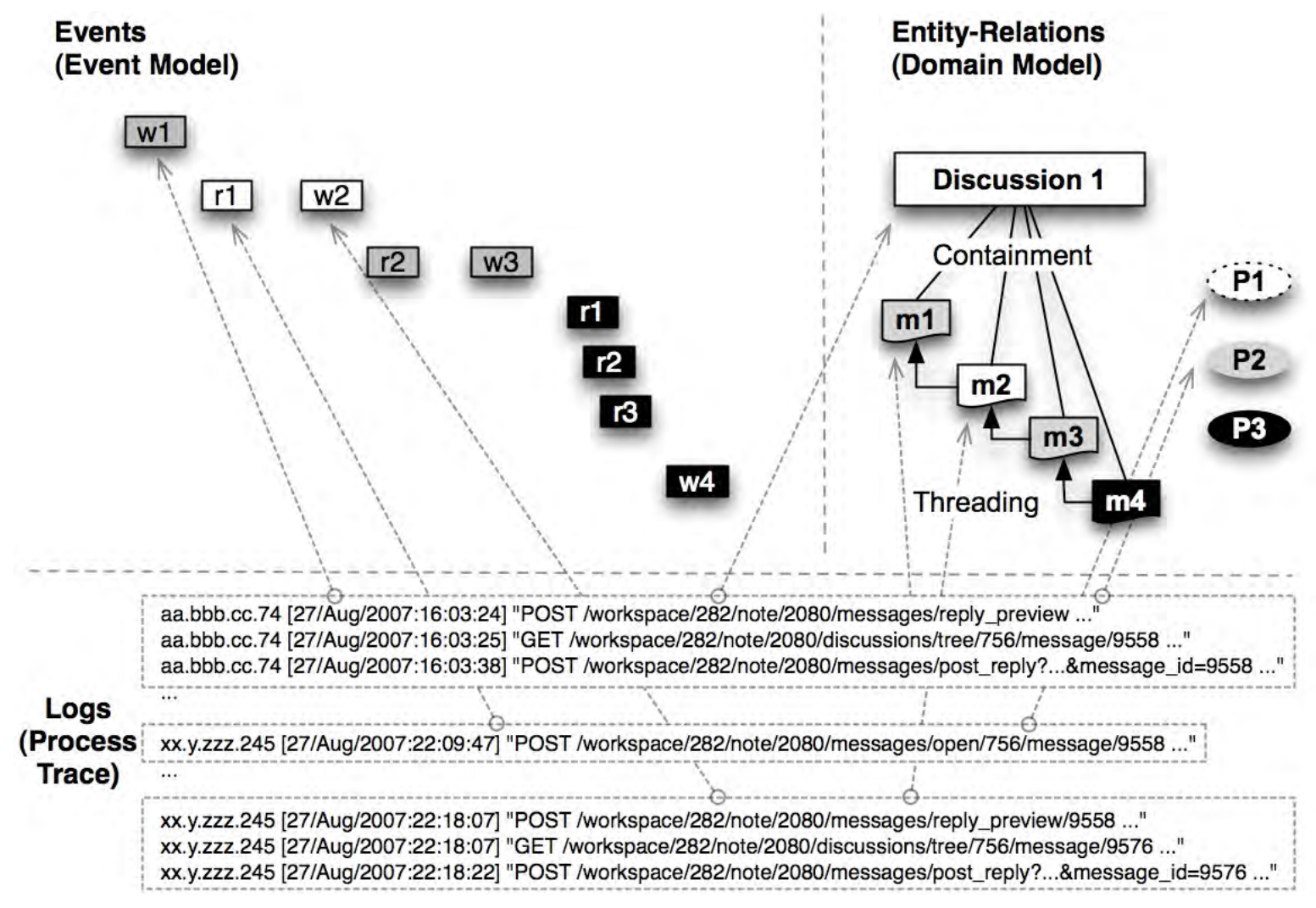

Figure 1. From Process Trace to Domain and Action Models

The lower portion of Figure 1 shows excerpts (edited for anonymity and simplicity of presentation) of a server log from one of our online community applications, disCourse [20]. ${ }^{1}$ The analytic hierarchy is illustrated throughout this paper by building on this simplified example.

\section{Entity-Relations: Domain Model}

Prior to or concurrently during the construction of the event model (next section), it is necessary to construct an ontology of the kinds of entities involved in the application domain of interest. Classes of entities and potential structural relationships between them are defined (e.g., actors, discussions, and messages, related by containment, threading and authoring relations). As the trace or log file is processed, new instances of entities and their structural relations are added to the domain model when they are encountered, along with relevant

\footnotetext{
${ }^{1}$ In our research, we use events logged by our software in a database. HTTP server logs of the same events are shown in this example to illustrate the method using log formats familiar to readers.
}

attributes that are expected to be needed for analysis. This is undertaken in conjunction with construction of the event model. For example, the right hand side of Figure 1 suggests how messages $\mathrm{m} 1, \ldots \mathrm{m} 4$ are created by participants P1, P2, P3 (shown by shading), related to each other by a threading relation, and contained in a discussion forum. The content of messages are also recorded in the domain model. Temporal information is recorded in the event model, discussed next.

\section{Events: Event Model}

The process trace is transformed into a set of events that constitute our first commitments concerning the relevant units for analyzing processes. This transformation involves the Exploratory Sequential Data Analysis (ESDA) operations of "chunking" and "coding" [17]. For example, the first three lines of the log of Figure 1 all are part of the process of posting a message in a system in which each message is previewed before posting. These three traces are chunked together and represented as the single event "w1" in the event model, along with information about the actor (P2, indicated by grey), 
action taken (w for writing), object (message $\mathrm{m} 1$ ), contents and location (recorded in the domain model), and temporal scope of the action. We call this layer the event model because the focus is on individual actions and other events by nonhuman actants such as software display events. The events have not yet been put in relation to each other.

Taken together, the event model and domain model provide an abstract transcript of the data in the process traces. If the transcription were complete with respect to the needs of a given analysis, then it would not be necessary to retain the original process traces. However, we retain pointers to the original process traces because a complete transcription is generally not possible. We may need to recover other information from the process trace, moving back and forth between the abstract transcript and the trace. Also, any transcript includes initial theoretical commitments [4, 15], which may turn out to be faulty, necessitating a return to the original process traces.

\section{Contingency Graphs: Contextualized Action Model}

Contingency graphs are an empirically grounded elaboration of the abstract transcript to make analytically relevant relationships between events explicit. We call these relationships contingencies because the relationships between events they capture may be merely contingent or incidental to the situation. The graph simply makes relationships that are latent in the data more explicit, and does not constitute a commitment concerning actors' intentions. Human action can be embedded in its context in many ways, including accidental relationships, or opportunistic leveraging of contextual and historical features as well as necessary antecedents for action [2, 9]. Thus, a contingency graph represents how action is embedded in the context of other events. Examples of contingency types we have used are listed in Table 2. A detailed presentation of the motivations and theory behind contingency graphs may be found in [21].

Clearly construction of a complete graph of the contingencies between events in a process trace is not practical, as it would result in a graph too complex for processing. (Imagine a graph in which each event is linked to every one involving the same actor, or the same object, or that has overlap in lexical content, or occurred nearby in time, and so on.) An analyst chooses those contingencies that are relevant for specific analytic purposes as guided by explicit or implicit theory. Therefore a contingency graph reflects further commitments on the part of the
Table 2. Examples of contingency types

\begin{tabular}{|c|c|}
\hline $\begin{array}{l}\text { Media } \\
\text { Dependency }\end{array}$ & $\begin{array}{l}\mathrm{e}_{\mathrm{i}} \text { operates on a media object or state } \\
\text { of that object that was created or } \\
\text { modified by } \mathrm{e}_{\mathrm{i}}\end{array}$ \\
\hline Same Actor & $\begin{array}{l}e_{i} \text { and } e_{j} \text { were due to acts of the same } \\
\text { actor }\end{array}$ \\
\hline \multirow[t]{2}{*}{$\begin{array}{l}\text { Inscriptional } \\
\text { Similarity }\end{array}$} & $\begin{array}{l}e_{i} \text { creates inscriptions with visual } \\
\text { attributes similar to those of } \\
\text { inscriptions created by } e_{j}\end{array}$ \\
\hline & $\begin{array}{l}\mathrm{e}_{\mathrm{i}} \text { creates inscriptions with lexical } \\
\text { strings identical to those in } \\
\text { inscriptions created by } \mathrm{e}_{\mathrm{j}}\end{array}$ \\
\hline Temporal & $\mathrm{e}_{\mathrm{i}}$ took place soon after $\mathrm{e}_{\mathrm{j}}$, where \\
\hline Proximity & $\begin{array}{l}\text { "soon" depends on the attentional } \\
\text { properties of the agents and } \\
\text { persistency of the medium }\end{array}$ \\
\hline $\begin{array}{l}\text { Spatial } \\
\text { Organization }\end{array}$ & $\begin{array}{l}\text { The locality of inscriptions operated } \\
\text { on in } e_{i} \text { is in a spatial context created } \\
\text { by } e_{j}(e . g . \text {. "grouping" a graphical } \\
\text { object by placing it near others) }\end{array}$ \\
\hline
\end{tabular}

analyst. However, even though a contingency graph is theoretically selective, we always base contingencies on empirically observable relationships between events found in the action and domain models, preferably those relationships that are unambiguous and can be detected automatically. If this standard of evidence is followed, a contingency graph can be treated as an abstract transcript that makes the evidence for interaction or other phenomena of interest manifest.

Contingency graphs can be constructed automatically from the layers below it [see, for example, 13]. For example, for each event in which an actor accessed an object we might scan back to find the last event in which the object's contents were modified, and install a media dependency. Contingencies can also be installed from a given event to the most recent prior event involving the actor, to prior events in which the actor accessed a media object with similar inscriptions (e.g., lexical phrases or graphical devices), or to temporally recent events in the same spatial site. A challenge with algorithmic installation of contingencies is limiting their number. Temporal or sequential proximity are useful (and computable) heuristics for selecting relevant contingencies, as they follow the local continuity of human attention and goal directed behavior.

For example, the lower half of Figure 2 shows the events of Figure 1 with contingencies installed. The single arcs represent media dependencies, and the double arcs represent multiple contingencies, 
such as temporal proximity combined with same actor and possibly inscriptional similarity. The act of reading a message $(\mathrm{r} 1, \mathrm{r} 2$, etc.) is media-dependent on the act of creating the message (w1, w2, etc.). The act of writing a message (e.g., w2) may be mediadependent on the act of creating the message to which it is a threaded reply (e.g., w1) and is contingent on the messages that the author has recently read (e.g., r1).

Once constructed, various kinds of analytic actions are possible on contingency graphs. For example, suppose a particularly productive session was identified in which participants made significant ideational progress. One option is to examine the interaction of the session participants more closely to identify the relationship between group processes and their accomplishments, and how participants appropriated the interactional affordances of the available media for these purposes. We have used the contingency graphs in several studies to support this kind of microanalysis of interaction [11, 12, 13, 22]. Recurring patterns of interaction so identified could be posed as hypotheses and searched for in the overall contingency graph to find other sessions that have similar patterns of activity, to see whether they display similar productivity. Another option is to look outside the session to find influences from or to other sessions. One can trace same-actor and mediadependency contingencies, following the actors and actants respectively. (Actant is Latour's [9] term for non-human entities that yet have agency in networks of associations.) Tracing proceeds forward in time to see whether the new ideas of the session were disseminated elsewhere, or backwards in time to identify possible predecessors of the ideational advance. Such an analysis would ground concepts of "bridges" and "brokers" in actual accomplishments, not relying solely on structural relationships that do not guarantee such accomplishments. At this writing we are constructing a contingency graph of several years of data from an online community of educators-Tapped-In [18, 5]-in preparation for application of methods such as those just described.

\section{Uptake Graphs}

As discussed above, contingencies are so named because they can include circumstantial relationships between acts with varying degrees of relevance to interaction. Analytic work is required to sort out the significance of contingencies and identify relationships between events that are not merely circumstantial, but reflect intentional acts. An act of uptake is one in which an actor takes traces of one or more prior events as having certain significance for

\section{Uptake Graph (Interaction Model)}

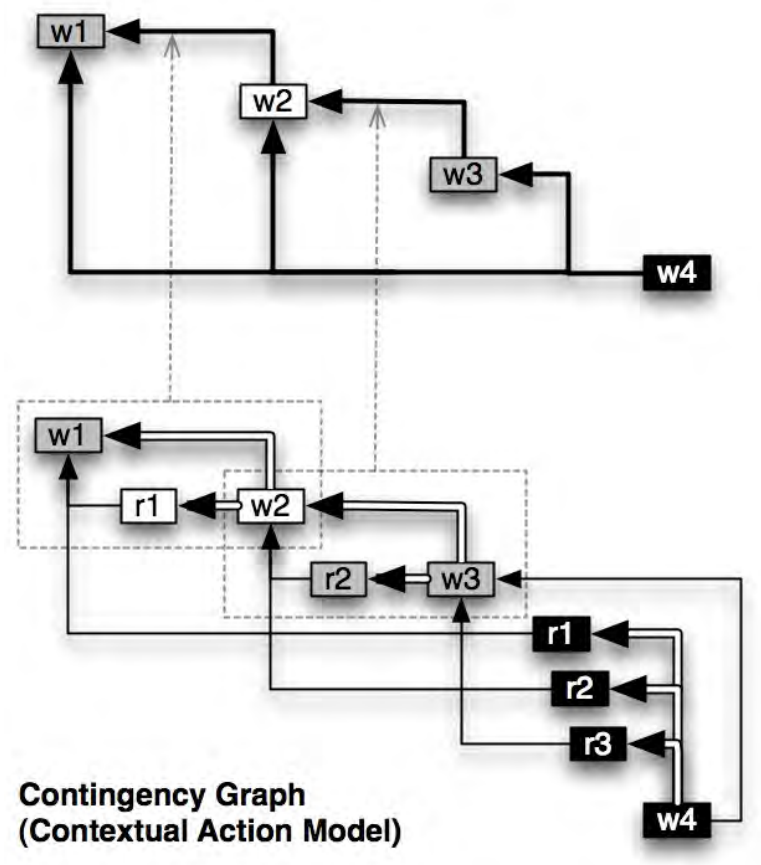

Figure 2. Contingency and Uptake Graphs

an ongoing activity [21]. For example, a speaker takes up some aspect of the prior speaker's utterance, or a message poster in a discussion forum can take up some aspect of the message being replied to. Uptake is a generalization of other analytic categories of interactional relationships, such as "reply" or "adjacency pair". It includes these relationshps, but also applies to spatio-temporally distributed associations between actors in which they may not even be aware of each other, let alone be directing their actions towards each other. Thus, uptake is more general than "transactivity" [1], which requires other-directedness.

We began our research focused on tightly coordinated interactions between dyads, but when we started studying asynchronous and distributed interaction we generalized our conception of interaction to other forms of association between actors, all of which have uptake in common. For example, a customer in Amazon.com may take up the recommendations of another customer, or be influenced by the histogram of ratings. The essential idea is that the trace one actor's actions have left in the environment (e.g., chat message, discussion posting, uploaded file, profile, recommendation) is taken up by another actor in some manner. Uptake of traces can result in stigmergic effects, i.e., implicit distributed coordination of collective action [16]. 
An uptake graph is an interaction model, as it describes the interaction that the analysis claims is taking place. Representationally, uptake is a collection of contingencies, as illustrated in Figure 2. An analyst collects contingencies that are considered to be analytically meaningful: a number of contingencies between two or more acts may corroborate the interpretation that the final act is an intentional taking up of traces of the prior ones. For example, w2, in which $\mathrm{P} 1$ posts a reply to the message posted by $\mathrm{P} 2$ in $\mathrm{w} 1$, is contingent on $\mathrm{w} 1$ in these ways: there is a media dependency ( $\mathrm{m} 2$ is linked by threading to $\mathrm{m} 1$ ); lexical overlap (m2 contains phrases also found in $\mathrm{m} 1$ ); and a chain of temporal proximity (w2 took place shortly after read event $\mathrm{r} 1$ by the same actor, and $\mathrm{r} 1$ is mediadependent on w1 by virtue of reading $\mathrm{m} 1$ ). All of these contingencies are taken as evidence for an intentional relationship of $\mathrm{w} 2$ to $\mathrm{w} 1$, and collapsed into one uptake arc. Because of this relationship between contingencies and uptake, an uptake graph may be seen as an abstraction of a contingency graph, and many of the same analytic moves (such as pattern matching and tracing actions) apply to both.

Contingency graphs were originally developed as an empirical offshoot of uptake graphs. We were constructing uptake graphs by hand in our analyses, and realized that we needed to record the evidence on which we based a judgment of the presence of uptake independently of that judgment. Although all analytic artifacts from process traces on up involve theoretical decisions, the move from contingency graphs to uptake graphs is a move from primarily empirically accountable representations to those more strongly determined by analytic interpretations.

Contingency and uptake graphs are described more fully in [21]. We have used contingency and uptake graphs to provide interactional accounts of specific accomplishments of participants [11, 12], to trace out information sharing [23], and to detect roles of participants not visible in the final media trace [22]. For example, examining only reply structure (the "threading" relationship between messages in Figure 1), we might miss the fact that $\mathrm{m} 4$ played an integrative role in this discussion (as is apparent in the uptake from all three prior messages in Figure 2)

Contingency and uptake graphs represent process models: they focus on how acts relate to each other and constitute a process of interaction. Their basic unit is acts and other events: the entities through which interaction takes place are hidden in the attributes of these events. Now we turn to a derived representation that makes these entities explicit.

\section{Associogram (Mediation Model)}

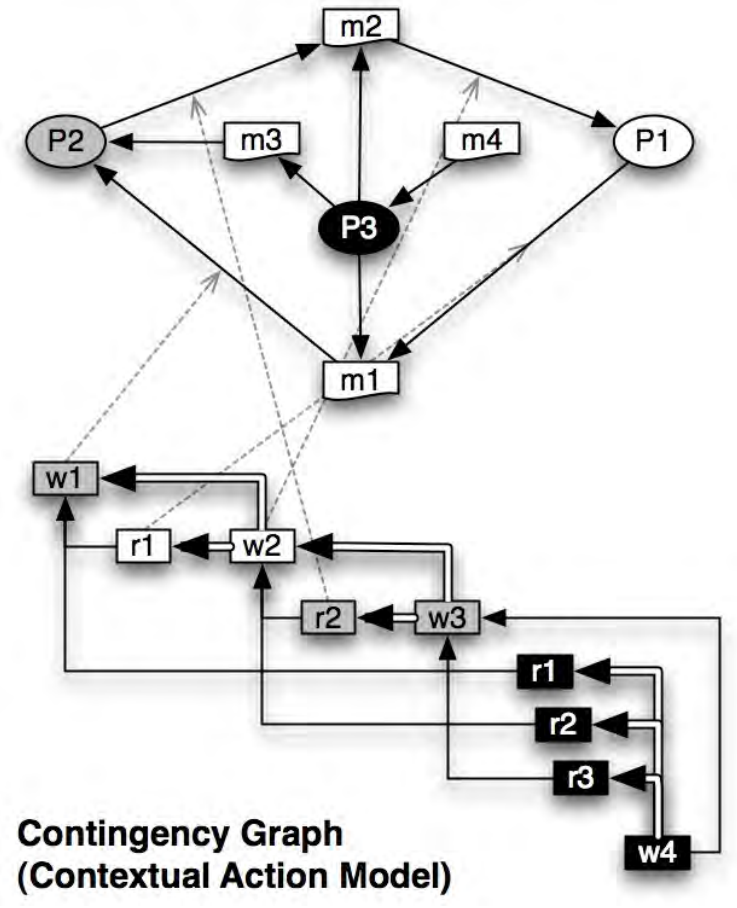

Figure 3. From Contingency Graph to
Associogram

\section{Associograms: Mediation Model}

In the study of socio-technical systems, we are interested in how the technological infrastructure enables and is utilized by the social actors to interact with each other. The next layer of the analytic hierarchy makes the objects of this technological infrastructure explicit and shows how they mediate interaction between participants. An analysis at this layer provides the mediation model, and is represented by multi-modal bipartite graphs ${ }^{2}$ in which participants are related to each other via the objects through which they interact. Directed arcs represent state-influence (a weaker form of state-dependency): they extend from an object to an actor if the state of the object is influenced by some action of the actor (e.g., writing a message or editing a wiki), and from the actor to the object if the state of the actor has been influenced by accessing the object (e.g., reading a message or wiki) We call these graphs associograms to distinguish them from sociograms in

\footnotetext{
${ }^{2}$ There may be more than two types of nodes, but they are divided into two partitions. Actors are in one partition and the various types of objects are in the other.
} 
a manner that honors Latour's (2005) concept of mediated associations that assemble a social system.

One can construct associograms from a set of events, whether taken directly from the event model, or events of interest that were selected from the contextualized action or interaction models (contingency graphs or uptake graphs, respectively). A node in any of these models represents an event, and actors and objects are attributes of the node. This is largely reversed in an associogram: actors and objects are nodes, and events are links between nodes. For example, in Figure 3, w1 - the event of P2 writing $\mathrm{m} 1$-becomes a directed association from $\mathrm{m} 1$ to P2 (m1's state depends on P1), and $\mathrm{r} 1$ - the event of $\mathrm{P} 1$ reading $\mathrm{m} 1$ - becomes a directed association from $\mathrm{P} 1$ to $\mathrm{m} 1$.

An associogram can be constructed at different granularities. Object nodes could be created for each individual object (e.g., one node for each message, wiki page, chat, etc.), or they could be aggregated for object types (e.g., all associations via messages aggregated into a single node, those via wikis in another, etc.). Some information is lost even in the former case: all the events involving an actor and an object will fall into the same two nodes and links between them. For example, if $\mathrm{P} 1$ reads $\mathrm{m} 1$ multiple times there is still only one link from P1 to $\mathrm{m} 1$, and if $\mathrm{P} 2$ edits a wiki multiple times, there is still one link from the wiki to $\mathrm{P} 2$. Some of this information can be preserved by annotating the links with number of occurrences, or by putting backpointers to the originating event nodes. Temporal sequencing is mostly lost, though it can be recovered by following these backpointers to the contingency graph. This information reduction is actually an advantage of associograms: they reduce the clutter of interaction models to expose recurring patterns of mediation. An example is given below, under "Finding Interaction Patterns." But first we consider how associograms can tell us about mediation.

\subsection{Characterizing Mediation}

Degree and path analysis of an associogram can reveal the roles different media play in a sociotechnical system. Media objects or media types (in an aggregate associogram) that have high in-degree are accessed by many actors, and hence may be influential sites and points where an intervention can reach many participants in a socio-technical system. Those with high out-degree are modified by many actors, and hence may be sites where ideas are aggregated or consolidated (potential roles as community memory, or locus of knowledge building). In a weighted associogram, heavily weighted links indicate that the incident objects are visited repeatedly by actors. These measures may be compared between different media types to assess their relative roles. For example, we have used associograms constructed from bridging events to assess the roles of different media (discussions, wikis, resources and profiles) in mediating bridging in a socio-technical system [20].

\subsection{Finding Interaction Patterns}

Associograms can help expose patterns of interest in contingency or uptake graphs. For example, consider the question of finding which participants are in dialogue with each other. A key indicator of the presence of dialogue might be what we call a "round trip": one participant makes a contribution that is accessed by another participant who then makes a contingent contribution (evidencing uptake) that the first participant then accesses [23]. In a contingency graph one would need to trace out many paths from each participant to find paths that go to another participant via a read and then a write and then back to the first participant. In an associogram one need only find cycles in the graph. If the links are weighted with frequency acounts, the minimum weight of the path could be taken as a measure of extent of dialogue. For example, in Figure 3 there is a cycle (following the arrows in reverse to trace chronology rather than dependency) $\quad \mathrm{P} 2 \rightarrow \mathrm{m} 1 \rightarrow \mathrm{P} 1 \rightarrow \mathrm{m} 2 \rightarrow \mathrm{P} 2$. This corresponds to the round trip in which $\mathrm{P} 2$ posts $\mathrm{m} 1$, $\mathrm{P} 1$ reads it and posts $\mathrm{m} 2$ in reply and $\mathrm{P} 2$ reads $\mathrm{m} 2$, completing the round trip. Note that P2 need not post a reply to $\mathrm{m} 2$ to compete the round trip: an analysis that looks only at the threading structure of posted messages and does not include read events would miss this round trip.

\subsection{Characterizing Mediated Relationships}

Associograms summarize the directionality of interaction between two people and the media through which this takes place. The subgraph of all paths of length two (direct mediation) between two
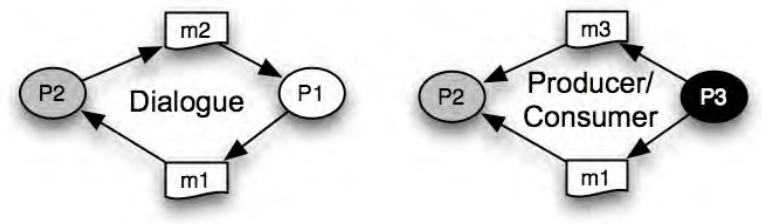

Figure 4. Pairwise Associations (Relationship Model) 
persons can be used in at least two ways to characterize the relationships between those persons as mediated by the socio-technical system. First, we can recognize defined patterns, as shown in Figure 4. Second, one might construct a vector of the weights on paths of different types (e.g., P1 to P2 via discussions, $\mathrm{P} 2$ to $\mathrm{P} 1$ via discussions, $\mathrm{P} 1$ to $\mathrm{P} 2$ via wikis, etc.), providing each pair of persons with a mediated interaction profile. Cluster analysis of these vectors might then reveal recurring types of relationships. These approaches are currently under investigation in a dissertation by Kar-Hai Chu.

\section{Sociograms: Affiliation Model}

Finally, we briefly note that associograms can be transformed to conventional sociograms by transitive closure of the paths between actors. As shown in Figure 5, this results in a directed graph (or an asymmetric matrix) representing the affiliations between actors. Well established methods of social network analysis can then be applied [24], but with advantages that would not be realized if one had merely constructed sociograms directly from source data (e.g., surveys about ties). A "tie" in a sociogram is really shorthand for a complex network of multimediated interactions that develop over time. If suitable back-pointers to prior representations (the associograms and, via them, the contingency graph) are maintained, then results obtained via social network analysis of a tie representation can then be interpreted and understood by expanding back to the mediation and interaction models underlying those ties. In fact, this unpacking of ties was one motivation for the development of this analytic hierarchy, a motivation elaborated below.

\section{Discussion}

At this writing, we have used contingency and uptake graphs extensively in manual analyses; we have implemented software tools for constructing contingency graphs automatically from log files and have developed some rudimentary analytic tools that leverage these representations; and we have used associograms in ad-hoc computational analyses. Currently funded work is developing a more comprehensive toolset that will be applicable to a variety of socio-technical systems. We continue to explore the capabilities of the analytic hierarchy.

Although this paper outlines how the analytic hierarchy is constructed as one goes from log data to more abstract representations of action, interaction, mediation and tie, it should be emphasized that the

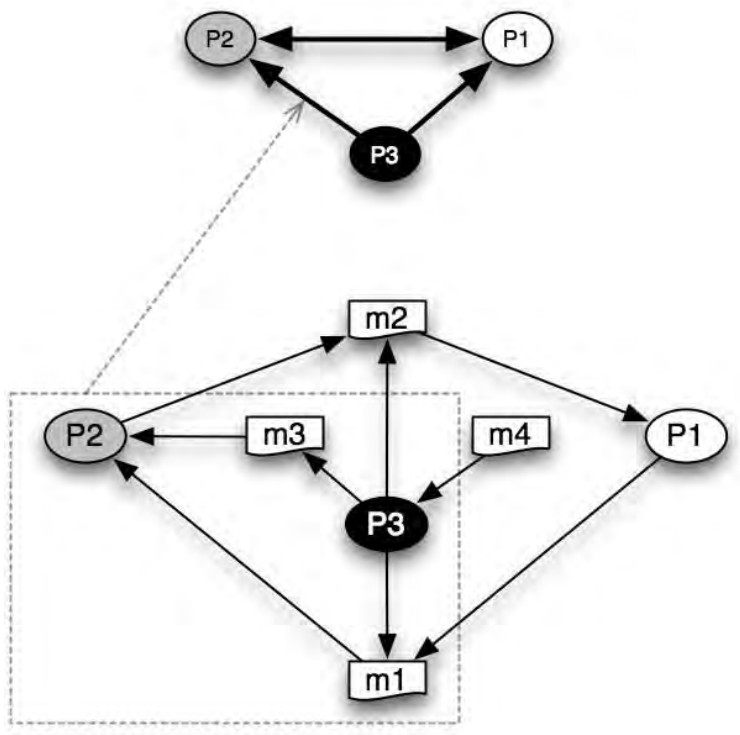

Figure 5. From Associogram to Sociogram

analytic hierarchy is not just a data interpretation framework. It is also intended to be a structural framework for connecting theorizing at different levels. Developmentally, the framework arose out of our own need to reconcile our research on small group interaction in computer supported collaborative learning and online learning contexts with our emerging research on online communities. It was clear that studies of communication networks, and social network analysis in particular, had something to offer, but the 'ties' of such analyses seemed to hide away the very processes we were interested in: the interaction and how it was influenced by and appropriated the media we were designing. Therefore we constructed the framework as an explicit bridge between analyses of local interaction and of larger social phenomena, with the expectation that it would also guide our bridging between theoretical explanations at these different levels.

This work is clearly sympathetic to calls by Contractor and colleagues [3, 14] for multitheoretical and multi-level (MTML) analyses and models of communication networks, but is based on a different conception of layering than MTML. The MTML approach calls for examining (1) the properties of individual nodes (incorporating attributed-based data); (2) properties of the network under consideration (including dyadic, triadic and global properties); and (3) relationships of this network to other relations over the same network constituents or the same relations as they change over time. These levels change the granularity or scope of analysis, but stay within an ontology of structural relations between a set of network constituents. Our 
hierarchical approach adds a more "vertical" dimension, changing the ontology between layers, from relationships between observed events, to mediated affiliations, to direct ties between actors.

Marin \& Wellman [10] contrast attribute based explanations, which explain behavior in terms of attributes of individuals, with the network analysis' position "that causation is not located in the individual, but in the social structure." Similarity of attributes is explained by similarity of network positions, due to the similar "constraints, opportunities and perceptions created by these similar network positions." We agree with this critique of attribute-based explanations but wish to avoid the opposite oversimplification: individuals' similarities do not arise merely out of static structures piping influences into the individuals from without. A range of thinkers, including Garfinkel [6], Blumer [2] and Latour [9] have argued (each in their own way) that social regularities are constructed and sustained through interaction between actors (whether strictly local interaction, as for Garfinkel, or potentially mediated across time and space, as for Latour). To fully understand social systems we must examine interaction. Colleagues ${ }^{3}$ have offered the analogy that ethnomethodological interaction is the quantum mechanics of social science. We can ignore it when explaining social life at a Newtonian level, but to really understand the origins of the social world we must dive in and find how fluctuations in microphenomena can have an influence on larger scale change. Latour [9] has made a similar observation in claiming that the "sociology of the social" may seem adequate for explanation of stable states of affairs, but Actor-Network Theory's "sociology of associations" is needed to understand changes in a social system.

Our position is that network structures are relevant because of how they support interaction: they are communication networks. "Communication networks" may reflect actual communication infrastructure that provide pathways through which interaction takes place; or they may only exist as researchers' representations that summarize the interactions that have taken place between actors without constituting any kind of enabling or constraining reality. In either case, and to continue the point concerning avoiding the opposing oversimplification, the network structure is not enough: to explain the origins of social life we must understand the nature of the communication or interaction that takes place. In socio-technical

\footnotetext{
${ }^{3}$ Ravi Vatrapu, personal communication, July 28, 2007; David Sallach , personal communication, May 23, 2010
}

systems, this includes understanding how that interaction is embedded in and exploits the resources of technological infrastructures; i.e., how it is mediated. The present work offers a conceptualization of how to map between these different levels of theory and analysis, viz., structure, mediation, and interaction; and also provides specific representations for supporting analytic work with computational tools. We hope that this framework will serve as a boundary object [19] for productive discourse between disciplines operating at these different levels.

\section{Acknowledgements}

This work was supported by NSF Award \#0943147. The view expressed herein do not necessarily represent the views of NSF. The author thanks Nathan Dwyer, Richard Medina, Ravi Vatrapu, Kar-Hai Chu and Devan Rosen for discussions through which the present framework was refined.

\section{References}

[1] M. W. Berkowitz and J. C. Gibbs, A Preliminary Manual for Coding Transactive Features of Dyadic Discussion, Ohio State University, 1979.

[2] H. Blumer, Symbolic Interactionism: Perspective and Method, University of California Press, Los Angeles, 1986.

[3] N. S. Contractor, S. Wasserman and K. Faust, Testing multi-theoretical multilevel hypotheses about organizational networks: An analytic framework and empirical example, Academy of Management Review, 31 (2006), pp. 681-703.

[4] A. Duranti, Transcripts, Like Shadows on a Wall, Mind, Culture \& Activity, 13 (2006), pp. 301310

[5] U. Farooq, P. Schank, A. Harris, J. Fusco and M. Schlager, Sustaining a community computing infrastructure for online teacher professional development: A Case Study of Designing Tapped In, Computer Supported Cooperative Work, 16 (2007), pp. 397-429.

[6] H. Garfinkel, Studies in Ethnomethodology, Prentice-Hall, Englewood Cliffs, New Jersey, 1967.

[7] R. Goldman, R. Pea, B. Barron and S. J. Derry, Video Research in the Learning Sciences, Lawrence Erlbaum Associates, Inc., Mahwah, NJ, 2007.

[8] B. Jordan and A. Henderson, Interaction Analysis: Foundations and practice, The Journal of the Learning Sciences, 4 (1995), pp. 39-103.

[9] B. Latour, Reassembing the Social: An Introduction to Actor-Network-Theory, Oxford University Press, New York, 2005. 
[10] A. Marin and B. Wellman, Social Network Analysis: An Introduction, in P. Carrington and J. Scott, eds., Handbook of Social Network Analysis, Sage, London, 2010.

[11] R. Medina, D. Suthers and R. Vatrapu, Inscriptions becoming representations, in $\mathrm{C}$. O'Malley, P. Reimann, D. Suthers and A. Dimitracopoulou, eds., Computer Supported Collaborative Learning Practices: CSCL 2009 Conference Proceedings, International Society of the Learning Sciences, Rhodes, Greece, 2009, pp. 18-27.

[12] R. Medina and D. D. Suthers, Bringing Representational Practice From Log to Light, in P. A. Kirschner, F. Prins, V. Jonker and G. Kanselaar, eds., International Perspectives in the Learning Sciences: Cre8ing a Learning World: Proceedings of the Eigth International Conference for the Learning Sciences (ICLS 2008), International Society of the Learning Sciences Utrecht, 2008, pp. 59-66.

[13] R. Medina and D. D. Suthers, Using $a$ contingency graph to discover representational practices in an online collaborative environment, Research and Practice in Technology Enhanced Learning, 4 (2009), pp. 281-305.

[14] P. R. Monge and N. S. Contractor, Theories of Communication Networks, Oxford University Press, Oxford, 2003.

[15] E. Ochs, Transcription as theory, in E. Ochs and B. B. Schieffelin, eds., Developmental Pragmatics, Academic Press, New York, 1979, pp. 43-72.

[16] D. Rosen and D. D. Suthers, Stigmergy and collaboration: Dynamic directed affiliation network analysis of mediated interaction, Proceedings of the Hawaii International Conference on the System Sciences (HICSS-44), January 4-7, 2010, Kauai, Hawai'i (CD-ROM), Institute of Electrical and Electronics Engineers, Inc. (IEEE), New Brunswick, 2011, pp. CD-Rom.

[17] P. Sanderson and C. Fisher, Exploratory sequential data analysis: Foundations, HumanComputer Interaction, 9 (1994), pp. 251-318.
[18] M. Schlager, J. Fusco and P. Schank, Evolution of an Online Education Community of Practice, in K. Renninger and W. Shumar, eds., Cambridge University Press, Building Virtual Communities, 2002, pp. 129-158.

[19] S. L. Star, The ethnography of infrastructure, American Behavioral Scientist, 43 (1999), pp. 377-391.

[20] D. D. Suthers and K.-H. Chu, Identifying mediators of socio-technical capital in a networked learning environment, in L. Dirckinck-Holmfeld, V. Hodgson, C. Jones, M. de Laat, D. McConnell and T. Ryberg, eds., Proceedings of the 7th International Conference on Networked Learning, Aalborg, Denmark, 2010, pp. 387-395.

[21] D. D. Suthers, N. Dwyer, R. Medina and R. Vatrapu, A framework for conceptualizing, representing, and analyzing distributed interaction, International Journal of Computer Supported Collaborative Learning, 5 (2010), pp. $5-42$.

[22] D. D. Suthers, N. Dwyer, R. Vatrapu and R. Medina, Analyzing Interactional Construction of Meaning in Online Learning, Proceedings of the 39th Hawai'i International Conference on the System Sciences (HICSS-39), January 4-7, 2006, Poipu, Kauai (CD-ROM), IEEE Computer Society Press, Poipu, Hawai'i, 2006.

[23] D. D. Suthers, R. Medina, R. Vatrapu and N. Dwyer, Information sharing is incongruous with collaborative convergence: The case for interaction, in C. Chinn, G. Erkens and S. Puntambekar, eds., The Computer Supported Collaborative Learning (CSCL) Conference 2007, International Society of the Learning Sciences, New Brunswick, 2007, pp. 714-716.

[24] S. Wasserman and K. Faust, Social Network Analysis: Methods and Applications, Cambridge University Press, New York, 1994. 